\title{
Oral Glucosamine Effect on Blood Glucose and Insulin Levels in Patients With Non-Diabetic Osteoarthritis: A Double-Blind, Placebo-Controlled Clinical Trial
}

\author{
Massoud SAGHAFI, ${ }^{1}$ Marjaneh KARIMI, ${ }^{2}$ Shokoufeh BONAKDARAN, ${ }^{3}$ Nadia MASSOUDNIA ${ }^{1}$ \\ ${ }^{1}$ Department of Rheumatology, Rheumatic Disease Research Center, School of Medicine, \\ Mashhad University of Medical Sciences, Mashhad, Iran \\ ${ }^{2}$ Department of Rheumatology, Masih Daneshvary Hospital, Shaheed Beheshti University of Medical Sciences, Tehran, Iran \\ ${ }^{3}$ Department of Endocrine, Endocrine Research Center, School of Medicine, Mashhad University of Medical Sciences, Mashhad, Iran
}

\begin{abstract}
Objectives: This study aims to determine the effects of oral glucosamine on glucose metabolism and insulin resistance in non-diabetic patients with osteoarthritis in Northeastern Iran.

Patients and methods: This placebo-controlled, randomized clinical trial included 40 non-diabetic patients with osteoarthritis (15 males, 25 females, mean age $63.8 \pm 7.64$ years; range 49 to 80 years). Patients were randomly divided into two equal groups and treated with oral glucosamine sulfate $1500 \mathrm{mg}$ a day or placebo for 90 days. Fasting blood sugar, glucose tolerance test with 75 grams glucose and serum insulin levels, and homeostatic model assessment-insulin resistance were evaluated initially and at the end of intervention.

Results: There were no significant differences between the groups in terms of blood sugar, glucose tolerance test, and insulin levels at the beginning and end of the study. In the oral glucosamine group, there were no significant changes in fasting blood sugar ( $94.1 \pm 7.14 \mathrm{mg} / \mathrm{dL}$ versus $93.5 \pm 9.45 \mathrm{mg} / \mathrm{dL}, \mathrm{p}=0.15)$, glucose tolerance test $(99.3 \pm 8.99 \mathrm{mg} / \mathrm{dL}$ versus $103.3 \pm 10.1 \mathrm{mg} / \mathrm{dL}, \mathrm{p}=0.07)$, and homeostatic model assessment-insulin resistance $(1.57 \pm 0.21$ versus $1.48 \pm 0.21, p=0.13)$ after treatment. Also, placebo did not significantly affect serum glucose levels and insulin resistance. Conclusion: Oral glucosamine with routine dosage was safe in our non-diabetic patients with osteoarthritis and had no significant effect on glucose metabolism and insulin resistance.

Keywords: Diabetes; glucosamine; glucose metabolism; insulin resistance; osteoarthritis.
\end{abstract}

Osteoarthritis (OA) is a very common disease in the elderly, affecting approximately $40 \%$ of patients older than 60 years of age. ${ }^{1}$ Analgesics and non-steroidal antiinflammatory drugs are conventional therapies in this regard, although with certain adverse effects. Popular and widelyused, glucosamines are currently administered for treating OA worldwide. On the other hand, type II diabetes mellitus is also more prevalent in this age population. In individuals aged over 50, OA may be concurrent with diabetes in $10-15 \%$ of the cases. ${ }^{2,3}$ Glucosamine may play a role in glucose metabolism, which has raised concerns about its safety profile in diabetic patients. The entry of glucosamine into cells is stimulated by insulin and involves the glucose-transporter system. Glucosamine is then phosphorylated to glucosamine- 6 phosphate by tissue hexokinases. ${ }^{4}$ Several animal and in vitro studies have shown that high doses of glucosamine can induce insulin resistance and raise plasma level of glucose..$^{5-8}$ However, other human studies having investigated shortterm intravenous glucosamine infusion effects did not report any such adverse impacts on either insulin sensitivity or glucose level..$^{9,10}$ 
Few studies aiming to evaluate the effects of oral standard doses of glucosamine in healthy humans concluded that oral glucosamine did not induce insulin resistance, ${ }^{11-13}$ these findings add fuel to the controversy of whether glucosamine can adversely affect glucose metabolism. In this study, we aimed to determine the effects of oral glucosamine on glucose metabolism and insulin resistance in non-diabetic patients with $\mathrm{OA}$ in Northeastern Iran.

\section{PATIENTS AND METHODS}

A total of 63 non-diabetic patients with OA (23 males, 40 females, mean age $64.8 \pm 8.66$ years; range 53 to 81 years) were initially registered for this randomized, double blind, placebo controlled clinical trial which was performed between February 2013 and May 2014 in the Rheumatology Department of Mashhad University of Medical Sciences, Mashhad, Iran. All patients met the American College of Rheumatology criteria for the diagnosis of $\mathrm{OA}$ of the knee or the hip.. ${ }^{14,15}$ Patients, either diagnosed with diabetes, or with a family history of diabetes, body mass index $<20$ and $>30 \mathrm{~kg} / \mathrm{m}^{2}$ ), history of liver or renal disease, myocardial infarction, inflammatory diseases, pregnancy or lactation, systemic diseases that induce insulin resistance and those taking drugs that interfere in glucose metabolism including anticonvulsants, glucocorticoids and antipsychotics, were excluded. Thirteen patients were excluded on the grounds that they either subsequently declined or failed to meet our inclusion criteria. The remaining 50 patients (18 males, 32 females, mean age 63.9 \pm 6.86 years; range 50 to 79 years) were divided into two equal groups in a blind manner by computerized randomization. Both observers and the patients were blind as to the type of randomization and the drug form. After drawing serum baselines, first group received the usual dose of $1500 \mathrm{mg}$ (500 mg x 3 capsules daily) glucosamine sulfate (Heaith Burst Company, Ohio, USA) for 90 days, whereas the second group received placebo capsules containing cellulose. Both groups were allowed to use analgesic medications, which they had taken before as needed. Every month, based on a random number chart, patients received boxes of 90 capsules of glucosamine or placebo from the dispenser in our center for three months and we ensured that the capsules were used fully and properly. After 90 days, laboratory tests were repeated in all patients. We excluded five patients in each group as they failed to take the drug or did not refer for follow-up. Finally, the study was completed with 40 patients (15 males, 25 females, mean age $63.8 \pm 7.64$ years; range 49 to 80 years) with non-diabetic $\mathrm{OA}$ and body mass index within the medium range.

Participants were informed about the potential side effects of glucosamine including abdominal discomfort, diarrhea, constipation and rising in blood glucose, lipid profiles and blood pressure. All patients gave informed consent for participation in the study. The study protocol was approved by the Ethics Committee of Mashhad University of medical sciences. This

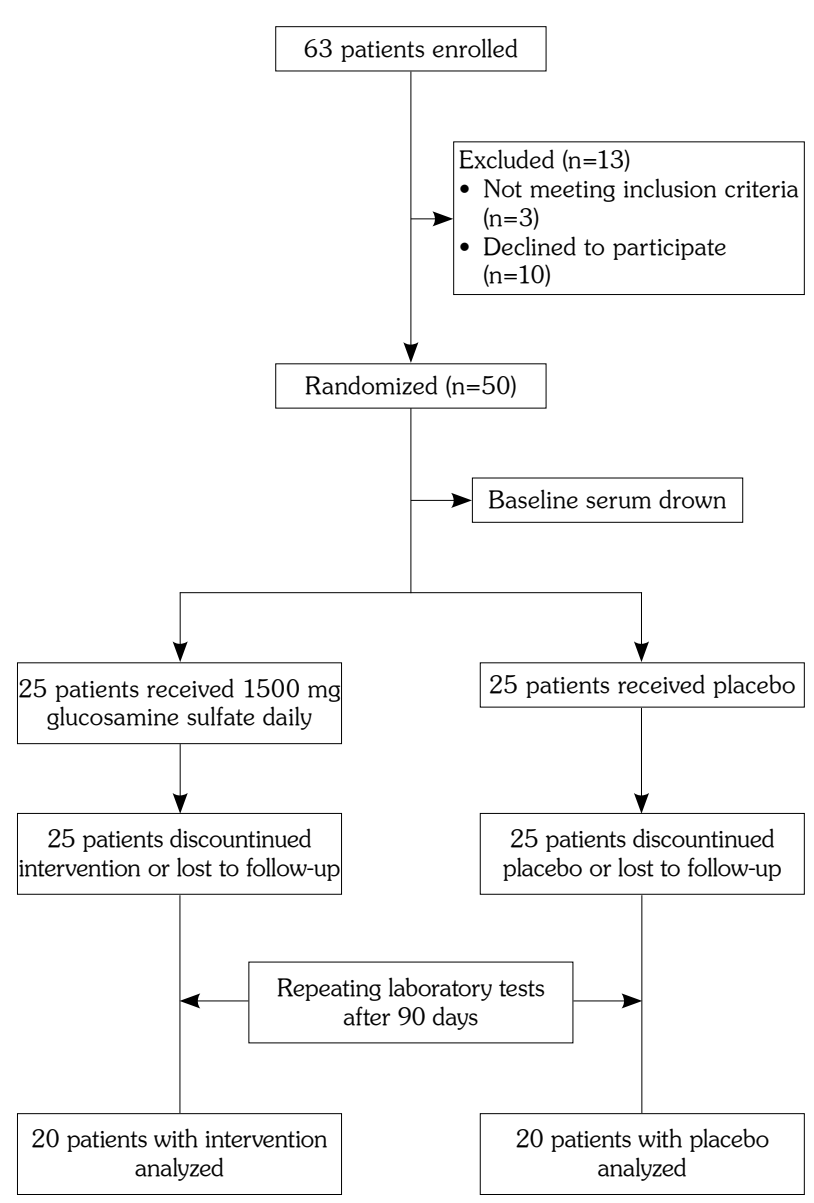

Figure 1. Consolidated standards of reporting trials flow diagram of this study. 
clinical trial was registered in IRCT (ID: IRCT 201304284167 N5). The study was conducted in accordance with the principles of the Declaration of Helsinki.

Following a thorough medical history as well as physical examination, baseline serum fasting blood sugar and insulin levels were measured for each subject, using glucose oxidase method (Human GmbH, Wiesbaden, Germany) and immunoradiometric assay with intra-assay coefficient of variation of $4.3 \%$ and inter-assay of $3.4 \%$, respectively. Then, patients underwent glucose tolerance test with 75 grams of glucose with blood glucose measured two hours later. Insulin resistance (Homeostasis Model Assessmentof Insulin Resistance=HOMA-IR ${ }^{16}$ ) was calculated as follows:

HOMA-IR $=\frac{\text { Fasting glucose }(\mathrm{mmol} / \mathrm{lit}) \times \text { insulin }(\mathrm{mlu} / \mathrm{mL})}{22.5}$

\section{Statistical analysis}

Laboratory results before and after the intervention were compared with paired student's t-test for normal distributed data. Wilcoxon signedrank test was used for the comparison of not normally distributed data. P-values less than 0.05 were considered significant. Consolidated standards of reporting trials flow diagram of the study is shown in Figure 1.

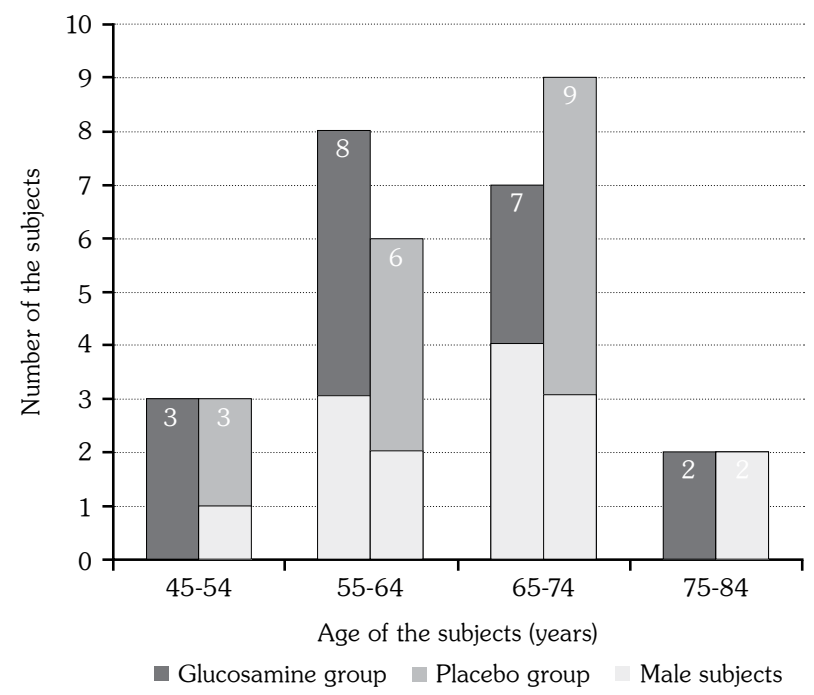

Figure 2. Demographic bars of two groups with their age $(p=0.44)$ and $\operatorname{sex}(p=0.74)$ distribution.

\section{RESULTS}

The two groups were similar in terms of mean age and sex distribution (Figure 2). The mean ages in the glucosamine and placebo groups were $63.4 \pm 7.74$ years and $64.9 \pm 8.46$ years, respectively, with no significant difference between the two $(p=0.44)$. Females constituted $65 \%$ and $60 \%$ of the glucosamine and placebo groups, respectively $(p=0.74)$.

There were no significant differences between the two groups regarding the levels of fasting blood sugar, insulin, HOMA-IR, and blood sugar after 75 grams load of glucose. There were no significant changes in the laboratory results after 90 days of intervention (Table 1). In the glucosamine group, there were no significant changes in fasting blood sugar $(94.1 \pm 7.14 \mathrm{mg} / \mathrm{dL}$ versus 93.5 $\pm 9.45 \mathrm{mg} / \mathrm{dL}, \mathrm{p}=0.15)$, glucose tolerance test $(99.3 \pm 8.99 \mathrm{mg} / \mathrm{dL}$ versus $103.3 \pm 10.1 \mathrm{mg} / \mathrm{dL}$, $\mathrm{p}=0.07)$, and homeostatic model assessmentinsulin resistance $(1.57 \pm 0.21$ versus $1.48 \pm 0.21$, $\mathrm{p}=0.13$ ) after treatment. Blood sugar, two hours after the consumption of 75 grams of glucose, increased slightly in the glucosamine group which was considered insignificant. Also, placebo did not significantly affect serum glucose levels and insulin resistance.

\section{DISCUSSION}

Osteoarthritis, a chronic degenerative disease, is considered to be one of the main causes of disability in the elderly population. Use of glucosamine, which is frequently prescribed for treating $\mathrm{OA}$, is still plagued with heated controversies regarding the potential effects on glucose metabolism. ${ }^{17,18}$

The effect of glucosamine on blood glucose and insulin sensitivity may be attributed to the inverse flux in the hexosamine pathway. ${ }^{19}$ Several experimental studies have been conducted to determine how it may affect glucose metabolism. ${ }^{4-7}$ These studies indicate high concentrations achieved via intravenous injection, which might impair insulin sensitivity and glucose tolerance. In a randomized-placebo controlled study, Monauni et al. ${ }^{10}$ concluded that high doses of glucosamine were in association with glucose tolerance impairments following intravenous glucose tolerance test. The authors concluded that 


\begin{tabular}{|c|c|c|c|}
\hline & Glucosamine group & Placebo group & \\
\hline & Mean \pm SD & Mean \pm SD & $p$ \\
\hline \multicolumn{4}{|c|}{ Fasting blood sugar (mg/dL) } \\
\hline Before & $94.1 \pm 7.14$ & $91.5 \pm 9.09$ & 0.23 \\
\hline After & $93.5 \pm 9.45$ & $97.6 \pm 8.99$ & 0.15 \\
\hline \multicolumn{4}{|c|}{ Blood sugar two hour after glucose $(\mathrm{mg} / \mathrm{dL})$} \\
\hline Before & $99.3 \pm 8.99$ & $101.0 \pm 11.3$ & 0.73 \\
\hline After & $103.2 \pm 10.01$ & $100.0 \pm 10.09$ & 0.32 \\
\hline \multicolumn{4}{|c|}{ Insülin $(\mu \mathrm{U} / \mathrm{L})$} \\
\hline Before & $6.8 \pm 0.99$ & $6.6 \pm 0.9$ & 0.71 \\
\hline After & $6.7 \pm 0.85$ & $7.0 \pm 0.95$ & 0.21 \\
\hline \multicolumn{4}{|l|}{ HOMA-IR } \\
\hline Before & $1.57 \pm 0.23$ & $1.48 \pm 0.21$ & 0.19 \\
\hline After & $1.48 \pm 0.21$ & $1.69 \pm 0.28$ & 0.13 \\
\hline
\end{tabular}

this result was possibly due to the effect of glucose inhibiting pancreatic glucokinase. Also, some investigators believe that glucosamine may affect serum glucose levels and insulin resistance at doses used to treat OA. ${ }^{20.21}$ On the other hand, several studies, including ours, indicate that usual doses of oral glucosamine have no significant effect on glucose metabolism. Our study was conducted in individuals with medium range of body mass index who suffered from OA and took oral glucosamine $1500 \mathrm{mg} /$ day for 12 weeks. Similarly, Muniyappa et al. $^{3}$ investigated the effects following the administration of $1500 \mathrm{mg} /$ day oral glucosamine with regard to insulin sensitivity and fasting blood glucose in both lean and obese patients, with no tangible alteration in plasma as well as glucose insulin levels in either group after six weeks. Other studies ${ }^{22-25}$ in diabetic patients showed that the administration of glucosamine $1500 \mathrm{mg} /$ day had no significant effect on diabetes related endpoints, concluding that glucosamine, at usual doses, does not have any adverse effect on glycemic control in diabetic patients.

In a review of 33 studies of oral glucosamine administration for an average of 17 weeks on 3,063 subjects, Anderson et al. ${ }^{26}$ concluded that there were no adverse effects on blood, urine or fecal parameters, neither any effect on glucose metabolism. Finally, a systematic review by Dostrovsky et al. ${ }^{27}$ demonstrated that clinical evidence regarding the effects of oral glucosamine on glucose metabolism does not seem consistent; thus further research should be warranted in this issue.
Given the disparity of the findings in this respect, we decided to conduct our clinical trial in a different area with rather different immunogenic background of patients with OA. Although most of our patients with $\mathrm{OA}$ receiving glucosamine reported partial relief of their symptoms, we did not aim to assess the efficacy of the drug in this study.

Inconsistent results in the previous studies may be attributed to their varying dosages, route and duration of glucosamine administration, and the heterogeneity in the state of glucose condition in patients with diabetes, impaired glucose metabolism, and normoglycemic conditions.

A limitation of this study was the use of the HOMA-IR model for assessment of insulin resistance. Although the glucose clamping technique is considered the gold standard for assessing insulin resistance, a close correlation between HOMA-IR and glucose clamping was shown in their estimation of insulin resistance. ${ }^{28}$ Another limiting factor was the relatively small sample size which limited the statistical results of our study.

In conclusion, oral glucosamine, when prescribed in routine doses in non-diabetic patients with OA, had no significant effect on blood glucose and insulin resistance. Further long-term studies using larger sample sizes with diabetic and non-diabetic patients with OA may shed more light on the topic. 


\section{Acknowledgement}

We would like to thank Ms. R. Paydar for her assistance in preparing the manuscript.

\section{Declaration of conflicting interests}

The authors declared no conflicts of interest with respect to the authorship and/or publication of this article.

\section{Funding}

The results described in this paper were parts of a medical thesis of Mashhad University of Medical Sciences which was supported by a grant (code: 87920) from vice chancellor for research, Mashhad University of Medical Sciences, Mashhad, Iran.

\section{REFERENCES}

1. National Collaborating Centre for Chronic Conditions (UK), editor. Osteoarthritis: National Clinical Guideline for Care and Management in Adults. London: Royal College of Physicians (UK); 2008.

2. Pham T, Cornea A, Blick KE, Jenkins A, Scofield $\mathrm{RH}$. Oral glucosamine in doses used to treat osteoarthritis worsens insulin resistance. Am J Med Sci 2007;333:333-9.

3. Muniyappa R, Karne RJ, Hall G, Crandon SK, Bronstein JA, Ver MR, et al. Oral glucosamine for 6 weeks at standard doses does not cause or worsen insulin resistance or endothelial dysfunction in lean or obese subjects. Diabetes 2006;55:3142-50.

4. Pouwels MJ, Jacobs JR, Span PN, Lutterman JA, Smits $\mathrm{P}$, Tack CJ. Short-term glucosamine infusion does not affect insulin sensitivity in humans. J Clin Endocrinol Metab 2001;86:2099-103.

5. Baron AD, Zhu JS, Zhu JH, Weldon H, Maianu L, Garvey WT. Glucosamine induces insulin resistance in vivo by affecting GLUT 4 translocation in skeletal muscle. Implications for glucose toxicity. J Clin Invest 1995;96:2792-801.

6. Biggee BA, Blinn CM, Nuite M, Silbert JE, McAlindon TE. Effects of oral glucosamine sulphate on serum glucose and insulin during an oral glucose tolerance test of subjects with osteoarthritis. Ann Rheum Dis 2007;66:260-2.

7. Wells L, Vosseller K, Hart GW. A role for $\mathrm{N}$-acetylglucosamine as a nutrient sensor and mediator of insulin resistance. Cell Mol Life Sci 2003;60:222-8.

8. Shankar RR, Zhu JS, Baron AD. Glucosamine infusion in rats mimics the beta-cell dysfunction of non-insulin-dependent diabetes mellitus. Metabolism 1998;47:573-7.

9. Stumpf JL, Lin SW. Effect of glucosamine on glucose control. Ann Pharmacother 2006;40:694-8.

10. Monauni T, Zenti MG, Cretti A, Daniels MC, Targher $\mathrm{G}$, Caruso B, et al. Effects of glucosamine infusion on insulin secretion and insulin action in humans. Diabetes 2000;49:926-35.

11. Yu JG, Boies SM, Olefsky JM. The effect of oral glucosamine sulfate on insulin sensitivity in human subjects. Diabetes Care 2003;26:1941-2.

12. Tannis AJ, Barban J, Conquer JA. Effect of glucosamine supplementation on fasting and non-fasting plasma glucose and serum insulin concentrations in healthy individuals. Osteoarthritis Cartilage 2004;12:506-11.

13. Biggee BA, Blinn CM, McAlindon TE, Nuite $M$, Silbert JE. Low levels of human serum glucosamine after ingestion of glucosamine sulphate relative to capability for peripheral effectiveness. Ann Rheum Dis 2006;65:222-6.

14. Altman R, Asch E, Bloch D, Bole G, Borenstein $\mathrm{D}$, Brandt $\mathrm{K}$, et al. Development of criteria for the classification and reporting of osteoarthritis. Classification of osteoarthritis of the knee. Diagnostic and Therapeutic Criteria Committee of the American Rheumatism Association. Arthritis Rheum 1986;29:1039-49.

15. Altman R, Alarcón G, Appelrouth D, Bloch D, Borenstein D, Brandt $\mathrm{K}$, et al. The American College of Rheumatology criteria for the classification and reporting of osteoarthritis of the hip. Arthritis Rheum 1991;34:505-14.

16. Chen CN, Chuang LM, Wu YT. Clinical measures of physical fitness predict insulin resistance in people at risk for diabetes. Phys Ther 2008;88:1355-64.

17. Pavelká K, Gatterová J, Olejarová M, Machacek S, Giacovelli G, Rovati LC. Glucosamine sulfate use and delay of progression of knee osteoarthritis: a 3-year, randomized, placebo-controlled, double-blind study. Arch Intern Med 2002;162:2113-23.

18. Reginster JY, Neuprez A, Lecart MP, Sarlet N, Bruyere $\mathrm{O}$. Role of glucosamine in the treatment for osteoarthritis. Rheumatol Int 2012;32:295967.

19. Robertson M, Dunshea F, Goddard M, Leury B. Stimulation of the hexosamine biosynthetic pathway by glucosamine in sheep. Asia Pac $\mathrm{J}$ Clin Nutr 2005; 14 (Suppl):S79

20. Biggee BA, Blinn CM, Nuite M, Silbert JE, McAlindon TE. Effects of oral glucosamine sulphate on serum glucose and insulin during an oral glucose tolerance test of subjects with osteoarthritis. Ann Rheum Dis 2007;66:260-2.

21. Pham T, Cornea A, Blick KE, Jenkins A, Scofield $\mathrm{RH}$. Oral glucosamine in doses used to treat osteoarthritis worsens insulin resistance. Am J Med Sci 2007;333:333-9.

22. Reginster JY, Deroisy R, Rovati LC, Lee RL, Lejeune $\mathrm{E}$, Bruyere $\mathrm{O}$, et al. Long-term effects of glucosamine sulphate on osteoarthritis progression: a randomised, placebo-controlled clinical trial. Lancet 2001;357:251-6.

23. Scroggie DA, Albright A, Harris MD. The effect 
of glucosamine-chondroitin supplementation on glycosylated hemoglobin levels in patients with type 2 diabetes mellitus: a placebo-controlled, doubleblinded, randomized clinical trial. Arch Intern Med 2003;163:1587-90.

24. Albert SG, Oiknine RF, Parseghian S, Mooradian AD, Haas MJ, McPherson T. The effect of glucosamine on Serum HDL cholesterol and apolipoprotein AI levels in people with diabetes. Diabetes Care 2007;30:2800-3.

25. Clegg DO, Reda DJ, Harris CL, Klein MA, O'Dell JR, Hooper MM, et al. Glucosamine, chondroitin sulfate, and the two in combination for painful knee osteoarthritis. N Engl J Med 2006;354:795-808.

26. Anderson JW, Nicolosi RJ, Borzelleca JF. Glucosamine effects in humans: a review of effects on glucose metabolism, side effects, safety considerations and efficacy. Food Chem Toxicol 2005;43:187-201.

27. Dostrovsky NR, Towheed TE, Hudson RW, Anastassiades TP. The effect of glucosamine on glucose metabolism in humans: a systematic review of the literature. Osteoarthritis Cartilage 2011;19:375-80.

28. George PS, Pearson ER, Witham MD. Effect of vitamin D supplementation on glycaemic control and insulin resistance: a systematic review and metaanalysis. Diabet Med 2012;29:142-50. 